\title{
Human hepatocellular adenoma as a promising cell source for bioartificial liver systems
}

\author{
QUN YAN $^{1,2}$, XIN-MEI ZHAO ${ }^{1}$, LI-JUAN DENG ${ }^{3}$, YU-XIN FANG $^{2}$, JIAN-JIAO LIN $^{1}$ and AI-MIN LI ${ }^{1}$ \\ ${ }^{1}$ Department of Gastroenterology, Longgang District People's Hospital of Shenzhen, Shenzhen, Guangdong 518116; \\ ${ }^{2}$ Department of Gastroenterology, Guangdong Provincial Key Laboratory of Gastroenterology, \\ Nanfang Hospital, Southern Medical University, Guangzhou, Guangdong 510515; ${ }^{3}$ Department of Gastroenterology, \\ Zhuhai Hospital of Traditional Chinese and Western Medicine, Zhuhai, Guangdong 519000, P.R. China
}

Received August 6, 2018; Accepted March 23, 2019

DOI: $10.3892 /$ etm.2019.7673

\begin{abstract}
The present study assessed human hepatocellular adenoma (HCA) as a potential source of biological material for bioartificial liver (BAL) systems. The histological characteristics of HCA tissues from 8 patients were examined using hematoxylin and eosin staining. The glycogen synthesis capacity of HCA cells was assessed using Periodic Acid-Schiff (PAS) staining and the expression of genes involved in liver function were examined using immunohistochemical staining (IHC) and reverse transcription-quantitative PCR analysis. Primary cells from HCA tissues were subsequently isolated and cultured in vitro. Cells within HCA tissues from 8 patients exhibited a polygonal shape, similar to that of cells in adjacent normal liver tissues. PAS staining of HCA tissues indicated the capacity of these cells to synthesize and store glycogen. Furthermore, IHC and PCR analyses revealed that the expression of liver function genes in HCA tissues were similar to those observed within normal adjacent liver tissues. Primary cells isolated from HCA tissues exhibited an irregular polygonal shape and positive in vitro growth. The current study demonstrated that HCA tissues exhibit histological and functional characteristics matching those of normal human liver tissue and may therefore be a promising alternative to hepatocytes as a source of biological material for BAL systems.
\end{abstract}

\section{Introduction}

A poor understanding of the pathophysiology of the condition, the heterogeneity among patient populations, the variety of possible etiologies and the lack of reliable outcome predictors

Correspondence to: Dr Ai-Min Li, Department of Gastroenterology, Longgang District People's Hospital of Shenzhen, 53 Aixin Road, Shenzhen, Guangdong 518116, P.R. China

E-mail: lam0725@163.com

Key words: bioartificial liver, hepatocellular adenoma, cell isolation, hepatic functions, liver failure means that the development of effective treatments for liver failure remains a major challenge (1). Without effective treatments, liver failure is associated with substantial physiological disability and a high mortality (2). Orthotopic liver transplantation has been demonstrated to be effective for the treatment of liver failure and the reduction of mortality (3). However, this strategy is greatly limited by a high cost and the lack of donor organs (4). Even when transplantation is feasible, a liver support system is compulsory to keep patients alive and maintain neurologic function until an appropriate liver is available (5).

The concept of a bioartificial liver (BAL) has emerged as a potential supportive therapy for patients with liver failure until transplantation can be performed or until recovery is achieved (6). A typical BAL system consists of hepatocytes and either a hollow fiber cartridge or semi-permeable membrane (bioreactor) (7). The efficacy of a BAL system was determined in a clinical study involving 24 patients with fulminant hepatic failure (FHF) (1). After treatment, 6 patients recovered without need for further transplantation, and in the other 18 patients, BAL provided an effective bridge treatment until transplantation (1). Human hepatocytes (autologous or allogeneic) are the optimal choice for the cellular component of BAL. However, the shortage of human livers and a lack of stable functional human hepatocyte lines remain critical obstacles that hinder the use of these cells in BAL systems (5). Porcine hepatocytes offer the advantage of being physiologically similar to human hepatocytes, readily available and easy to isolate (8). More importantly, porcine hepatocytes tend to form cell aggregates, which have been revealed to exhibit a beneficial effect on the maintenance of hepatic function (9). Demetriou et al (10) reported improved survival in patients with fulminant hepatic failure (FHF) or subfulminant hepatic failure who were treated with an extracorporeal porcine hepatocyte-based BAL. The use of xenogeneic cells is, however, associated with induced immunogenicity as well as the transmission of zoonoses including as endogenous porcine retroviruses (11).

Hepatocellular adenoma (HCA) is an uncommon benign liver tumor, which is linked to the use of hormonal contraceptives (12). Research has indicated that the monoclonal properties of HCA may facilitate cell lineage establishment 
and storage (13), making HCA an attractive potential source for the cellular component of the BAL system. Therefore, the present study was performed to assess the feasibility of using HCA as a cell source for BAL development.

\section{Materials and methods}

HCA specimen collection. The use of human liver samples for scientific research was approved by the Medical Ethics Committee of Nanfang Hospital, Southern Medical University (Guangzhou, China) and all enrolled subjects provided their written informed consent. HCA tissues were obtained from 8 patients who underwent liver hepatectomies at the Department of Hepatobiliary Surgery of Nanfang Hospital (Guangzhou, China) between September 2008 and March 2013. Adjacent liver tissue, which was located at least $2 \mathrm{~cm}$ away from the lesion, was collected from the same patients for use as control tissue. All patients had been diagnosed with adenomatous hyperplasia based on pathological analysis and did not receive any other treatment relevant to this study prior to surgery. Patient characteristics are recorded in Table I. The tissues used in the present study exhibited histopathological features that were typical of HCA. The HCA tumors were soft and well demarcated with little or no fibrous capsule, and were composed of liver cell plates that were mildly thickened or irregular. Thin-walled arteries supplied the tumor parenchyma without other portal tract elements including significant connective tissue, bile ducts or ductular reaction. Hepatocytes within the HCA tumors were of normal size with cytoplasm that was normal, clear or fatty and some lesions were almost entirely steatotic.

Prior to tissue dissociation, two small tissue fragments were removed. One fragment was prepared for hematoxylin and eosin (H\&E), Periodic Acid-Schiff (PAS) and immunohistochemical (IHC) staining by fixation in neutral buffered formalin (10\%) for $24 \mathrm{~h}$ at room temperature, paraffin embedding, sectioning (4 $\mu \mathrm{m}$ thickness), deparaffinization and dehydration via a series of graded ethanol rinses. The second tissue portion was immersed in RNA stabilization solution (Ambion; Thermo Fisher Scientific, Inc.) and cryopreserved at $-80^{\circ} \mathrm{C}$ for RNA extraction.

$H \& E$ staining. Dried sections of HCA tissue samples were processed according to the standard H\&E staining procedure (14). In brief, sections were stained with hematoxylin (Zhongshan Goldenbridge Biotechnology Co., Ltd.) for $10 \mathrm{~min}$ followed by differentiation in a hydrochloride acid-alcohol mixture. Then the sections were counterstained in eosin (Zhongshan Goldenbridge Biotechnology Co., Ltd.) for $3 \mathrm{~min}$, dehydrated in ethanol and cleaned in xylene before examination using a light microscope (Olympus BX40; magnification, $\mathrm{x} 400)$.

PAS staining. Dried sections of HCA tissue were also stained using a PAS staining kit (Fuzhou Maixin Biotech Co., Ltd.) according to manufacturer's protocol. The PAS-stained sections were counterstained with hematoxylin for $6 \mathrm{~min}$ at room temperature and differentiated in a hydrochloride acid-alcohol mixture, followed by blue color development in PBS, dehydration in ethanol, cleaning in xylene and examination using light microscopy (magnification, x200).
Table I. HCA patient characteristics.

Characteristic

Mean age, years (range)

$40(22-58)$

Sex, $\mathrm{n}(\%)$

Male

Female

Tumor size, $\mathrm{n}(\%)$

$\leq 5 \mathrm{~cm}$

$>5 \mathrm{~cm}$

Number of tumors, $\mathrm{n}(\%)$

$\leq 1$

$>1$

ICH and immunocytochemical staining. Prior to ICH or immunocytochemical staining, dried sections of HCA tissue were heated in a microwave oven (1000 watts for $10 \mathrm{~min})$ in $0.01 \mathrm{M}$ sodium citrate buffer ( $\mathrm{pH}$ 6.0) for $20 \mathrm{~min}$ and washed with PBS for antigen retrieval. Endogenous peroxidase activity was subsequently terminated by incubation in $3 \% \mathrm{H}_{2} \mathrm{O}_{2}$ solution for $20 \mathrm{~min}$ at room temperature. Tissue sections were then incubated at $4^{\circ} \mathrm{C}$ overnight with the following specific primary antibodies: Goat anti-human albumin (ALB) monoclonal antibody (cat. no. sc-46293; 1:100; Santa Cruz Biotechnology, Inc.), goat anti-human cytokeratin 18 (CK18) monoclonal antibody (cat. no. sc-31700; 1:50; Santa Cruz Biotechnology Inc.) and rabbit anti-human cytochrome p450 2E1 (CYP2E1) monoclonal antibody (cat. no. PB0186; 1:50; Wuhan Boster Biological Technology, Ltd.). Incubation with biotin-conjugated goat anti-rabbit IgG (cat. no. PV-9001; Zhongshan Goldenbridge Biotechnology Co., Ltd.) and biotin-conjugated rabbit anti-goat IgG secondary antibodies (cat. no. PV-9003; 1:500; Zhongshan Goldenbridge Biotechnology Co., Ltd) was performed after the samples were maintained at room temperature for $45 \mathrm{~min}$. The antibody-antigen complexes were visualized with diaminobenzidine (1:20, Zhongshan Goldenbridge Biotechnology Co.,Ltd) and sections were counterstained with hematoxylin for $3 \mathrm{~min}$ at room temperature. Finally, the sections were dehydrated in ethanol, cleared in xylene and examined under light microscopy (magnification, $\mathrm{x} 200)(15)$.

The IHC slides were analyzed by three independent investigators. Each sample was given a score according to the intensity of the staining ( 0 , no staining; 1 , weak staining; 2 , moderate staining; and 3, strong staining) and the extent of stained cells $(0,<5 \% ; 1,5-25 \% ; 2,26-50 \% ; 3,51-75 \%$; and $4,76-100 \%)$. The percentage of positive cell staining for each intensity was multiplied by the corresponding intensity value to obtain an immunostaining score ranging from 0 to 12 . Products of the positive staining scores and color intensity scores ranging from $0,1-4,5-8$, and $9-12$ were finally considered as negative staining $(-)$, slight positive staining $(+)$, medium positive staining $(++)$, and strong positive staining (+++), respectively.

Reverse transcription-quantitative (RT- $q$ ) PCR analysis. Cryopreserved HCA tissues were ground in a mortar and Trizol ${ }^{\circledR}$ 
Table II. Sequences of primers used for RT-qPCR analysis.

Primer sequences

\begin{tabular}{lll}
\cline { 2 - 3 } Primer & \multicolumn{1}{c}{ Forward } & \multicolumn{1}{c}{ Reverse } \\
\hline ALB & 5'-GCCTGCTGACTTGCCTTCATTAG-3' & 5'-TCAGCAGCAGCACGACAGAGTA-3' \\
TTR & 5'-CAGAAAGGCTGCTGATGACA-3' & 5'-ATGCCAAGTGCCTTCCAGTA-3' $^{\prime}$ \\
AAT & 5'-CAACCTGGCTGAGTTCGCCT-3' & 5'-CTCGCTGAGGAACAGGCCAT-3' \\
TF & 5'-CCCTTAACCAATACTTCGGCTAC-3' & 5'-TTTGCCAAGTTCTCAAATATAGTCG-3' \\
CYP2E1 & 5'-CTACAAGGCGGTGAAGGAA-3' & 5'-TCTCATTGCCCTGTTTCCC-3' \\
G-6-P & 5'-GCTGCTCATTTTCCTCATCAA-3' & 5'-TTCTGTAACAGCAATGCCTGA-3' \\
GST- $\pi$ & 5'-CCTGTACCAGTCCAATACCATCCT-3' & 5'-TCCTGCTGGTCCTTCCCATA-3' \\
CPS-1 & 5'-TGAGGGATGCTGACCCCATT-3' & 5'-CATTGTTGGCGTTGAGCCAG-3' \\
TAT & 5'-AGGCCAGGTGGTCTGTGAGG-3' & 5'-AGGGGTGCCTCAGGACAGTG-3' \\
CYP3A5 & 5'-CCTTACCCCAGTTTTTGAAGCA-3' & 5'-TCCAGATCAGACAGAGCTTTGTG-3' \\
CYP3A4 & 5'-CAGGAGGAAATTGATGCAGTTTT-3' & 5'-GTCAAGATACTCCATCTGTAGCACAGT-3' \\
GST- $\alpha$ & 5'-TGGCAGAGAAGCCCAAGCTC-3' & 5'-TGCACCAGCTTCATCCCATC-3' \\
AFP & 5'-GCTGACATTATTATCGGACAC-3' & 5'-GAACTTGTCATCAGAGAATGC-3' \\
CK-18 & 5'-GAGACGTACAGTCCAGTCCTTGG-3' & 5'-CCACCTCCCTCAGGCTGTT-3' \\
HNF-1 $\alpha$ & 5'-TACACCACTCTGGCAGCCACACT-3' & 5'-CGGTGGGTACATTGGTGACAGAAC-3' \\
GAPDH & 5'-GAAGGTGAAGGTCGGAGT-3' & 5'-GAAGATGGTGATGGGATTTC-3' \\
\hline
\end{tabular}

reagent (Takara Bio, Inc.) was used to extract RNA according to the manufacturer's protocol. RT of RNA generated $60 \mu \mathrm{l}$ cDNA which contained $1.8 \mu \mathrm{g}$ RNA, $12 \mu \mathrm{l}$ 5X PrimeScript ${ }^{\mathrm{TM}}$ buffer, $3 \mu$ 1 PrimeScript ${ }^{\mathrm{TM}}$ reverse transcriptase (RTase), $3 \mu 1$ random primers, $3 \mu \mathrm{l} \mathrm{dNTP}$ and RNase-free water (added to achieve a total volume of $60 \mu \mathrm{l}$ ) using a reverse transcription reagent kit (Takara Bio, Inc.). The reaction was sustained at $37^{\circ} \mathrm{C}$ for $15 \mathrm{~min}$. RTase was heat-inactivated at $85^{\circ} \mathrm{C}$ for $5 \mathrm{sec}$. Gene expression was measured using a RT-qPCR system (LightCycler ${ }^{\circledR}$ Real Time PCR; Roche Diagnostics). The reaction solution volume was $20 \mu \mathrm{l}$ and included forward and reverse primers (each, $0.2 \mu \mathrm{M}$; as indicated in Table II), $10 \mu \mathrm{l}$ SYBR Green, $7.2 \mu 1$ sterile distilled water and $2 \mu \mathrm{l}$ cDNA. The reactions were performed as follows: 40 cycles of $95^{\circ} \mathrm{C}$ for $30 \mathrm{sec}$ and $60^{\circ} \mathrm{C}$ for $20 \mathrm{sec}$ followed by one cycle of $65^{\circ} \mathrm{C}$ for $15 \mathrm{sec}$. Relative gene expression levels were calculated using the $2^{-\Delta \Delta C q}$ method with GAPDH (Invitrogen; Thermo Fisher Scientific, Inc.) as the endogenous control gene (16).

Isolation and culture of human HCA cells. Human HCA cells were isolated from the fresh surgically resected specimens by collagenase digestion as described previously (17), with minor modifications in collagenase type and centrifugation time. A HCA tumor fragment was perfused through vascular orifices on cut surfaces with Hank's Balanced Salt Solution (HBSS; Gibco; Thermo Fisher Scientific, Inc.) at $37^{\circ} \mathrm{C}$ for $15 \mathrm{~min}$ to remove blood cells. The fragment was subsequently perfused with a collagenase type IV solution (diluted in HBSS to a concentration of $200 \mathrm{U} / \mathrm{ml}$; Gibco; Thermo Fisher Scientific, Inc.) for a further $15 \mathrm{~min}$, and HCA cells were released by mincing the tissue and repeatedly pipetting the solution with a large-bore pipette. The cell suspension was filtered through a sterile $100 \mu \mathrm{m}$ nylon mesh, sedimented by centrifugation at $50 \mathrm{x} \mathrm{g}$ for $5 \mathrm{~min}$ at room temperature, resuspended and washed twice with cold HBSS solution. The isolated HCA cells were seeded at a density of $1 \times 105 / \mathrm{cm}^{2}$ of viable cells $(80 \%$ were determined to be viable following Trypan blue staining for $5 \mathrm{~min}$ ) in DMEM (Invitrogen; Thermo Fisher Scientific, Inc.) on collagen-coated culture plates (Invitrogen) supplemented with $10 \%$ fetal calf serum (HyClone; GE Healthcare Life Sciences), insulin (10-8 M; Sigma-Aldrich; Merck KGaA) and dexamethasone (10-8 M; Sigma-Aldrich; Merck KGaA) for culture at $37^{\circ} \mathrm{C}$ in $5 \% \mathrm{CO}_{2}$. Growing cell colonies were individually picked and amplified. The medium was changed twice a week and cell passages were performed with $0.25 \%$ trypsin.

Statistical analysis. All statistical analyses were performed using SPSS 19.0 software (IBM Corp.). The difference of clinical diagnostic scores between adjacent liver tissues and hepatocellular adenoma, based on ICH staining, was analyzed using Wilcoxon rank sum test. The difference of gene expression between the two independent groups was calculated using a Student's t test. $\mathrm{P}<0.05$ was determined to indicate a statistically significant result.

\section{Results}

Characteristics of HCA lesions. A total of 13 tumors were observed in 8 patients. The mean tumor size was $4.36 \pm 1.38 \mathrm{~cm}$. Images of the HCA and adjacent liver tissues are presented in Fig. 1A and B. HCA tissues collected from patients with adenomatous hyperplasia exhibited reddish brown coloring similar to that of the adjacent liver tissue. Histological examination of the HCA specimens revealed cords of hepatocytes, similar in appearance to normal hepatic parenchyma but lacking portal tracts, bile ducts and hepatic veins. Hepatocytes in the HCA samples exhibited a polygonal shape, similar to that of 
A

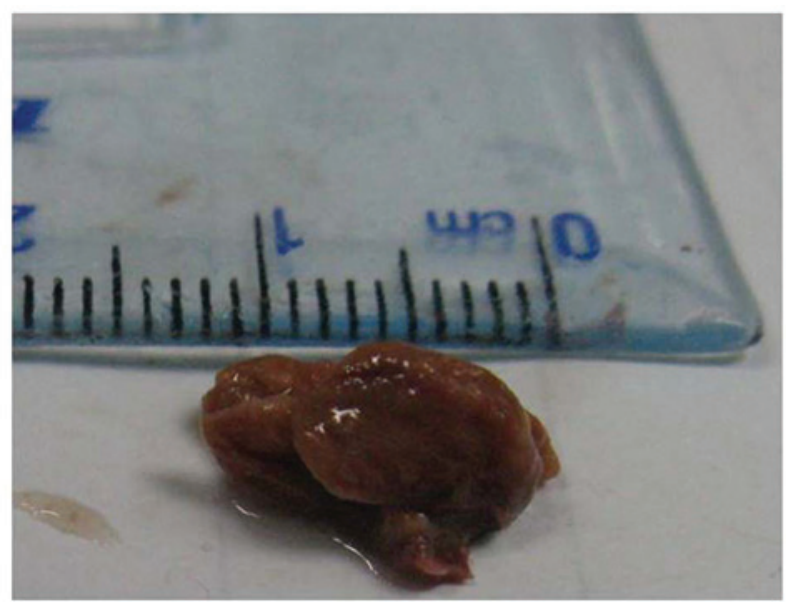

$\mathrm{C}$

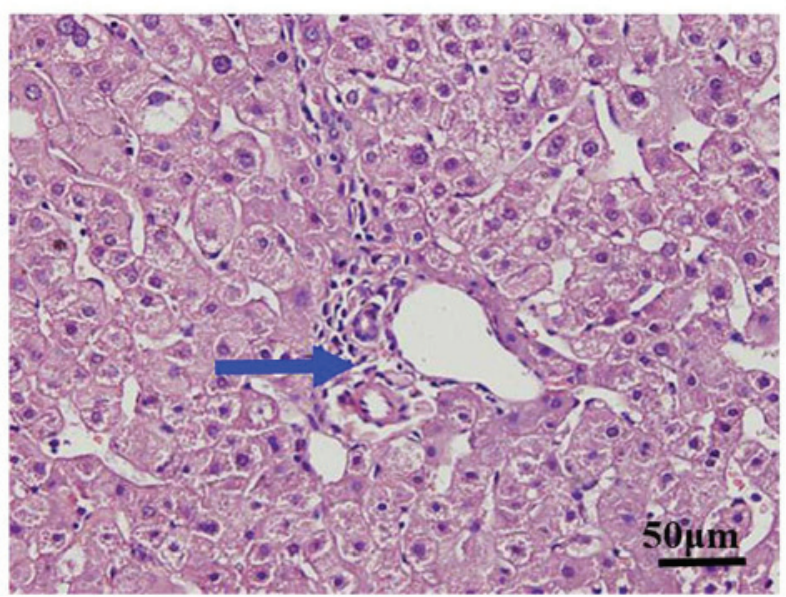

$\mathrm{B}$

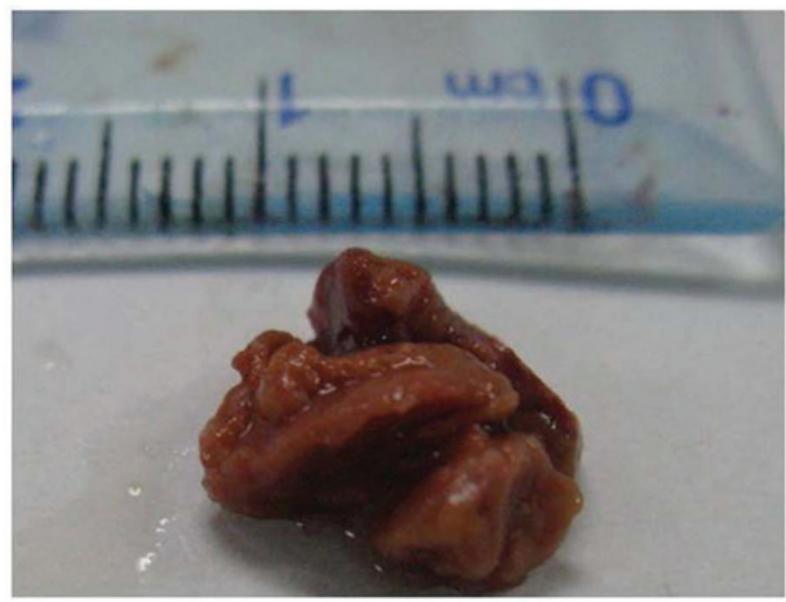

D

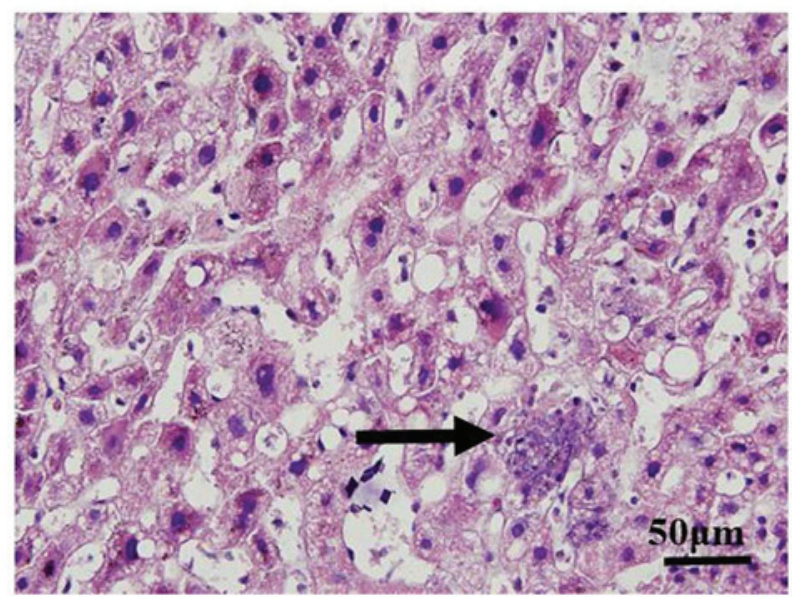

Figure 1. Representative digital photographs of extracted (A) adjacent liver tissues and (B) HCA passage 1 cells. The isolated cells extracted from different patients revealed a similar appearance. Representative images of histological staining of (C) adjacent liver tissues and (D) HCA tissue. The blue arrow indicates the presence of a portal triad and the black arrow indicates inflammatory cell infiltration. HCA, hepatocellular adenoma.

hepatocytes in adjacent liver tissues (Fig. 1C). Excessive lipid accumulation and inflammatory cell infiltration was observed in only the HCA tissues (Fig. 1D).

As presented in Fig. 2A and B, HCA and adjacent liver tissues stained positively with PAS, indicating the presence of glycogen. Stained hepatocytes in adjacent liver tissues revealed homogeneous intensity, whereas in HCA tissues, the distribution of stained cells was heterogeneous with varying intensity.

Hepatic function of HCA tissue. To assess the hepatic function of HCA tissue, the expression of ALB, CK18 and CYP2E1 was examined by IHC and compared with normal liver tissues (Fig. 3). HCA and adjacent liver tissues exhibited homogeneous cytoplasmic staining for ALB, CK18 and CYP2E1. The quantitative results in Table III reveal that resected specimens from four of the eight HCA cases $(50 \%)$ exhibited strong ALB staining and the other four cases exhibited slight-to-medium ALB staining. All HCA specimens exhibited medium-to-strong CK18 staining. Following CYP2E1 staining, four of eight HCA specimens $(50 \%)$ exhibited medium positive staining, whereas the other half exhibited slight positive staining. Statistical analysis revealed that the expression of ALB, CK18 and CYP2E1 in HCA samples did not differ significantly from that of the normal liver tissues ( $\mathrm{P}=0.574 ; 0.442 ; 0.721$, respectively).
Based on RT-qPCR analysis, the expression of synthetic function-specific genes [ALB, transthyretin (TTR), $\alpha$-1-antitrypsin (AAT) and transferrin (TF)] were similar or higher in the HCA group compared with those in adjacent liver tissues (Fig. 4). Similar results were observed for genes associated with detoxification including CYP2E1, CYP3A5 and CYP3A4. In addition, the expressions of genes related to metabolism and conversion function including glutathione S-transferase- $\alpha$ (GST- $\alpha)$, carbamoyl-phosphate synthase 1 (CPS-1), tyrosine aminotransferase (TAT) and glutathione S-transferase- $\pi$ (GST- $\pi$ ) demonstrated relatively large differences between groups. However, only the expression of GST- $\pi$ demonstrated statistical difference between groups, and was decreased in HCA tissues compared with adjacent liver tissues ( 0.38 vs. $0.53 ; \mathrm{P}=0.032$ ), likely due to the limited sample quantity. For liver phenotype markers, CK18 and hepatocyte nuclear factor $1 \alpha$ were expressed at similar levels in HCA and normal tissues, whereas $\alpha$ fetoprotein (AFP) expression was increased in HCA tissues compared with the control tissues (0.013 vs. 0.008; $\mathrm{P}=0.047$ ). These results are in agreement with those of IHC staining and the subsequent scoring analysis.

Isolation and culture of primary HCA cells. Based on the aforementioned function tests, the current study attempted to isolate primary cells from HCA via two-step collagenase 

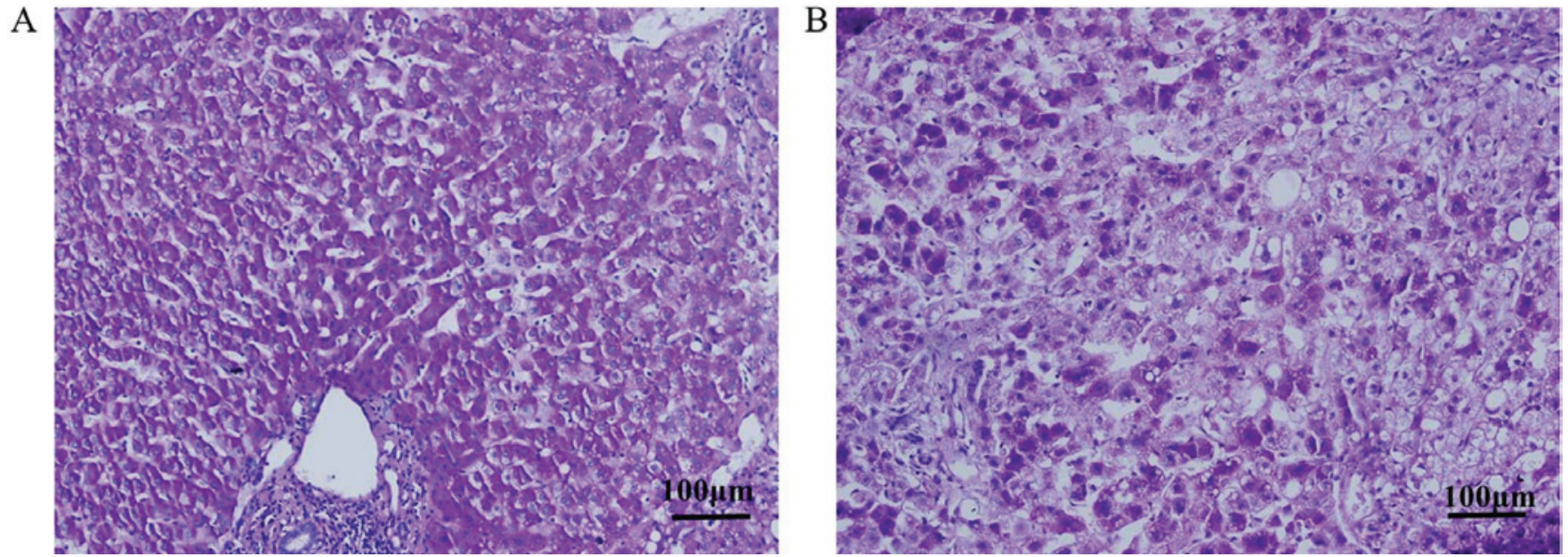

Figure 2. Representative images of periodic acid-schiff staining of (A) adjacent liver tissues and (B) human hepatocellular adenoma tissue.
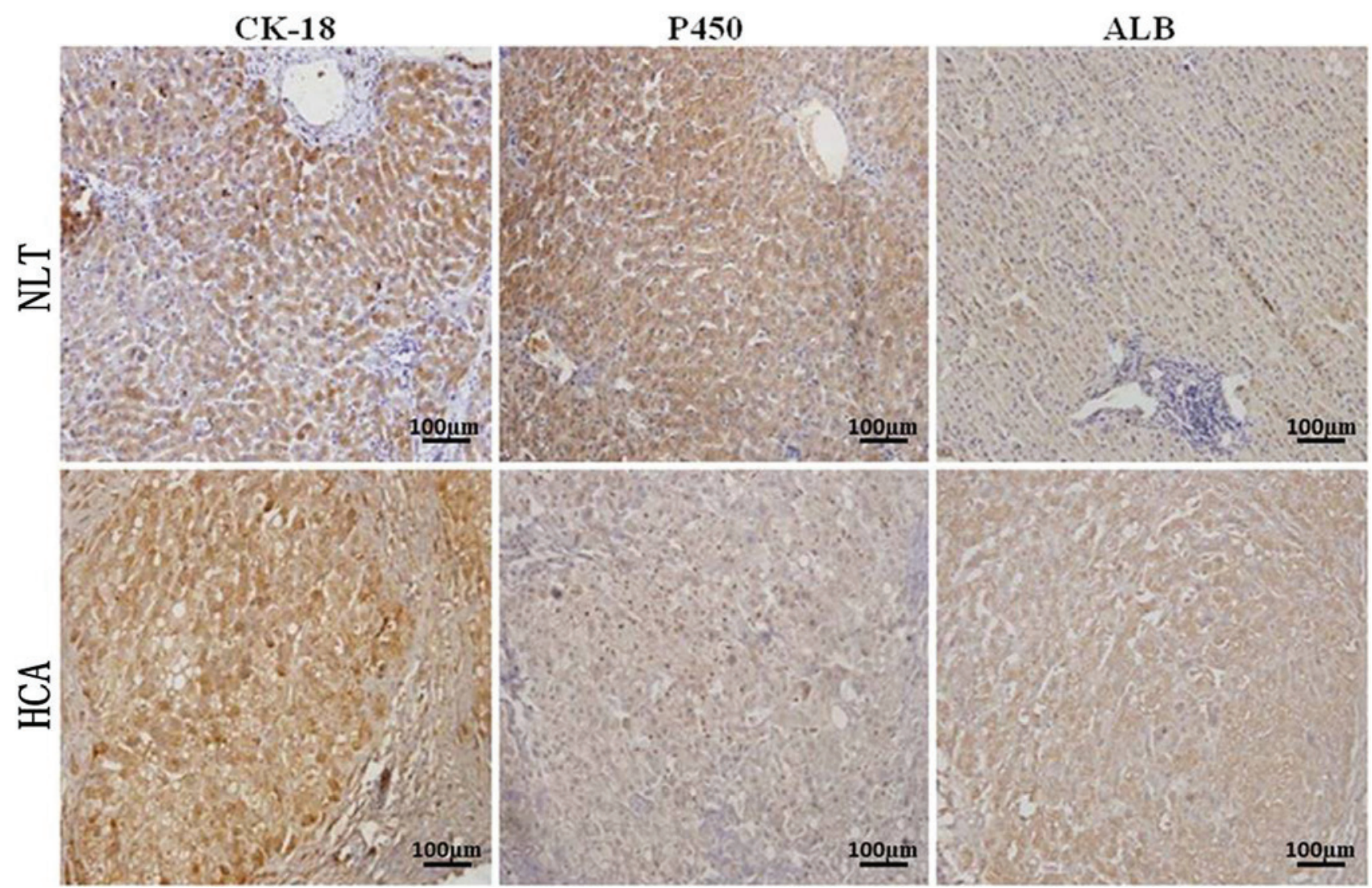

Figure 3. Immunohistochemical staining of adjacent liver tissues (upper panel) and human hepatocellular adenoma tissues (lower panel) with goat anti-human ALB monoclonal antibody (1:100), goat anti-human CK18 monoclonal antibody (1:50), and rabbit anti-human CYP2E1 (P450) monoclonal antibody (1:50). ALB, albumin; CK18, cytokeratin-18; CYP2E1, cytochrome p450.

perfusion. Most of the collected tissues, including adjacent normal and HCA tissues, were subjected to cell isolation. All normal cells and most of the isolated HCA primary cells did not proliferate in vitro and underwent apoptosis within several weeks (data not shown). A few HCA primary cells could be passaged 2-5 times. As a result, the current study hypothesizes that HCA primary cells may be more proliferative than normal cells. In Fig. 5, micrographs demonstrate the most proliferative isolated HCA primary cells following $>10$ passages that were isolated from a 22-year-old female patient pathologically diagnosed with HCA. Initially, the isolated primary hepatocytes grew slowly (Fig. 5A and B). After several weeks, most primary cells underwent apoptosis, while a continuously proliferating cell colony was observed (data not shown). The cells individually grew into cell colonies and the colonies were further picked by trypsin digestion for amplification due to the higher proliferative potential. The isolated HCA cells gradually gained active proliferative capacity and exhibited spindle cell morphology (Fig. 5C). These cells appeared to be smaller in size than normal hepatocytes after adhesion in vitro (Fig. 5D). 
Table III. Clinical diagnostic scores based on immunohistochemical staining.

\begin{tabular}{|c|c|c|c|c|c|c|}
\hline Protein & Tissues & - & + & ++ & +++ & P-value \\
\hline \multirow[t]{2}{*}{ ALB } & Adjacent liver tissues & 0 & 0 & 3 & 5 & \multirow[t]{2}{*}{0.574} \\
\hline & Hepatocellular adenoma & 0 & 1 & 3 & 4 & \\
\hline \multirow[t]{2}{*}{ P450 } & Adjacent liver tissues & 0 & 2 & 6 & 0 & \multirow[t]{2}{*}{0.442} \\
\hline & Hepatocellular adenoma & 0 & 4 & 4 & 0 & \\
\hline \multirow[t]{2}{*}{ CK18 } & Adjacent liver tissues & 0 & 0 & 3 & 5 & \multirow[t]{2}{*}{0.721} \\
\hline & Hepatocellular adenoma & 0 & 0 & 2 & 6 & \\
\hline
\end{tabular}

The score represents the product of the positive staining score and color intensity score. Positivity percentages of $<5 \%, 5-25 \%, 26-50 \%, 51-75 \%$ and $>75 \%$, were scored as $0,1,2,3$, and 4 , respectively. Color intensities corresponding to no coloring, slight yellowish, yellow-brownish, and dark brownish were scored as $0,1,2$, and 3, respectively. Products of the positive staining scores and color intensity scores of $0,1-4,5-8$, and 9-12 were finally considered as negative staining (-), slight positive staining $(+)$, medium positive staining (++), and strong positive staining $(+++)$, respectively. ALB, albumin; CK-18, cytokeratin -18 .
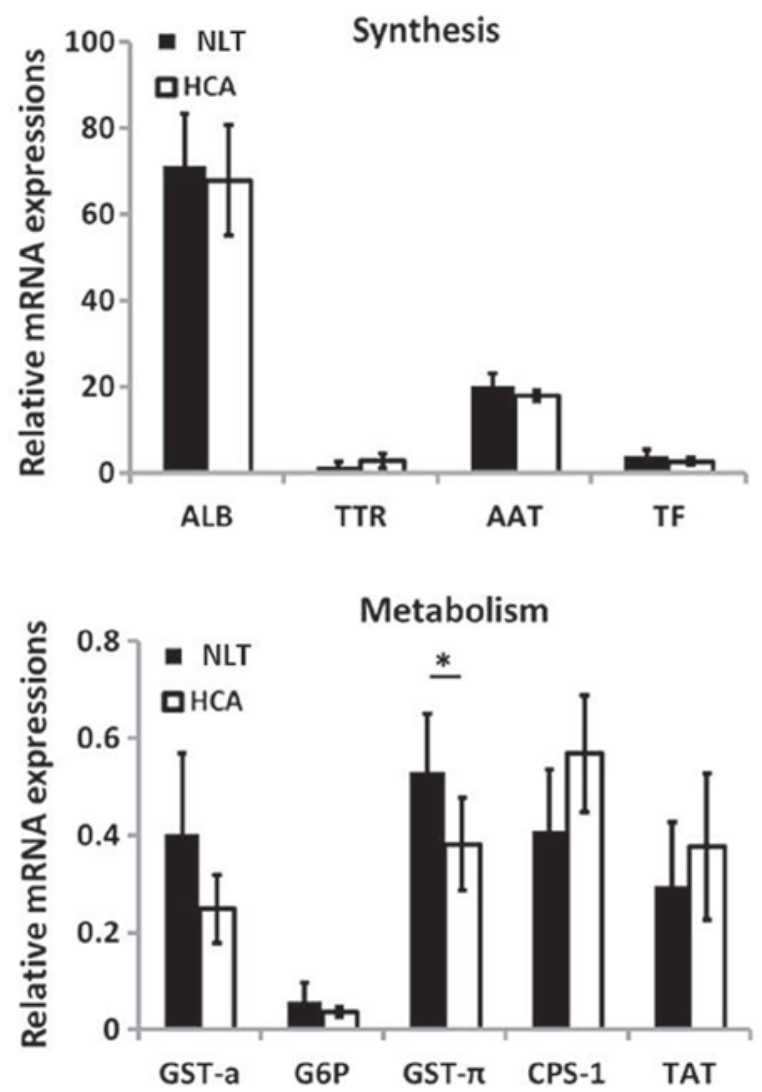

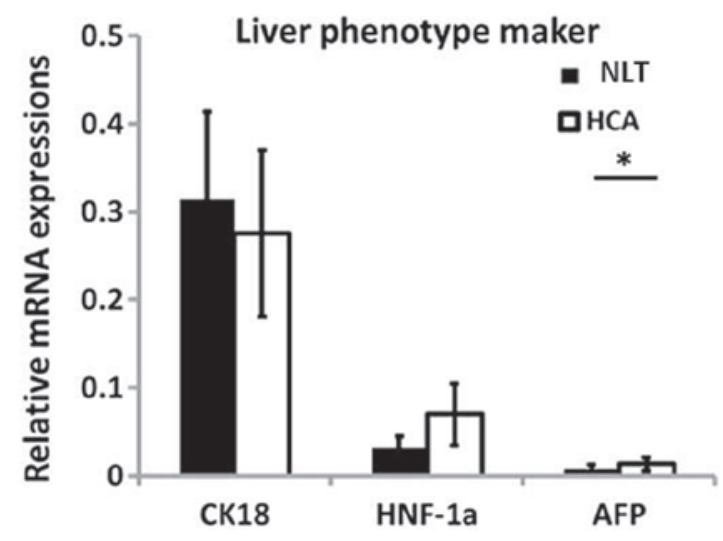

Detoxication

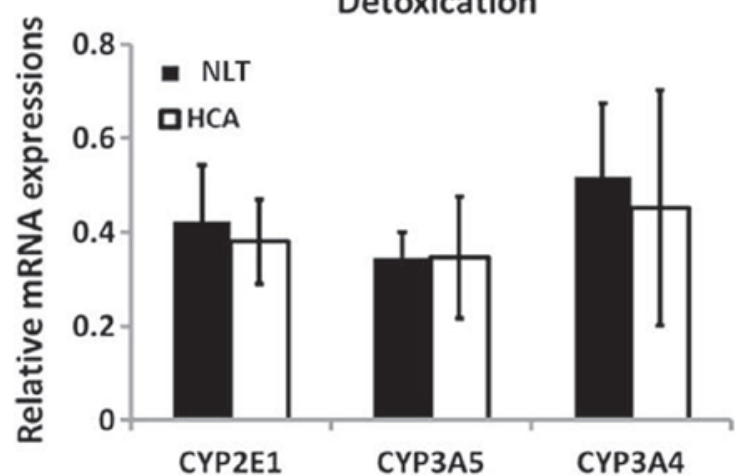

Figure 4. Comparison of hepatic function-specific gene expression between HCA and adjacent liver tissues. Most gene expression levels in the HCA group were comparable with those in the control group except the expression of GST- $\pi$ and AFP. *P<0.05. HCA, hepatocellular adenoma; ALB, $\alpha$ fetoprotein; NLT, normal liver tissue; TTR, transthyretin; AAT, $\alpha$-1-antitrypsin; TF, transferrin; HNF-1 $\alpha$, hepatocyte nuclear factor 1 homeobox A; G6P, glucose-6-phosphate; CPS-1, carbamoyl-phosphate synthase 1; TAT, tyrosine aminotransferase; CYP, cytochrome.

\section{Discussion}

HCA is a rare monoclonal benign liver tumor, which originates in the epithelium and usually develops within a healthy liver (18). HCA is more prevalent in females than in males and the use of oral contraceptives and anabolic steroids are established risk factors for the disease (19). Recent research has focused on the etiology, clinical manifestations and diagnosis of HCA (19). However, its functionality as a liver-derived tumor is rarely characterized. In the present study, the hepatic function of resected HCA tissues was assessed and the feasibility of HCA-derived cells as cellular components of BAL systems was determined.

The liver is a vital organ with a variety of important functions in the human body including detoxification, amino acid and lipid metabolism, protein and urea synthesis, and waste removal (20). Liver failure (acute or chronic) significantly compromises the aforementioned functions, resulting in a high 
A

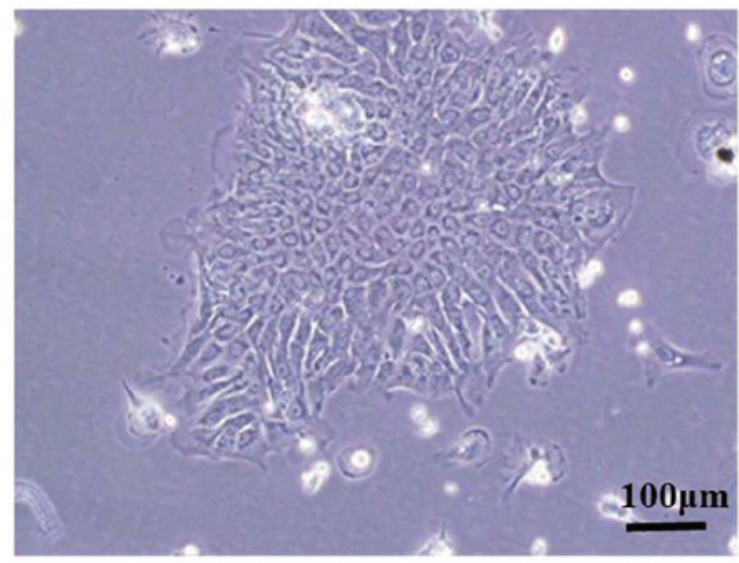

$\mathrm{C}$

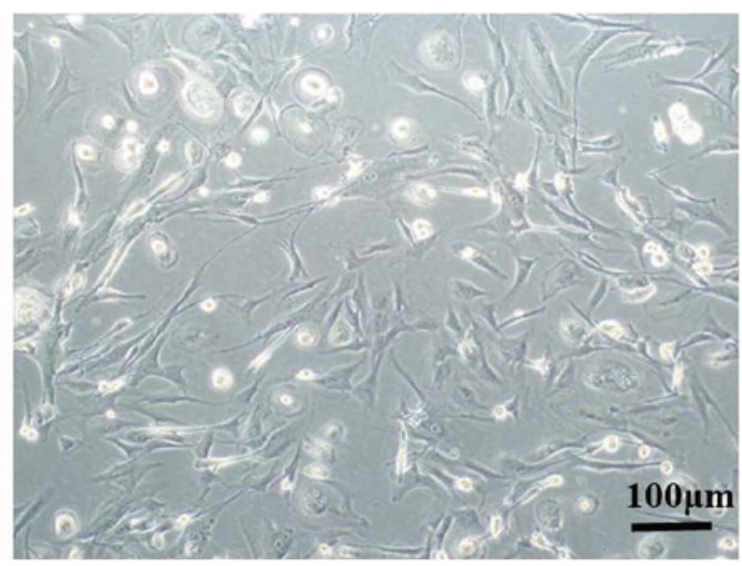

B

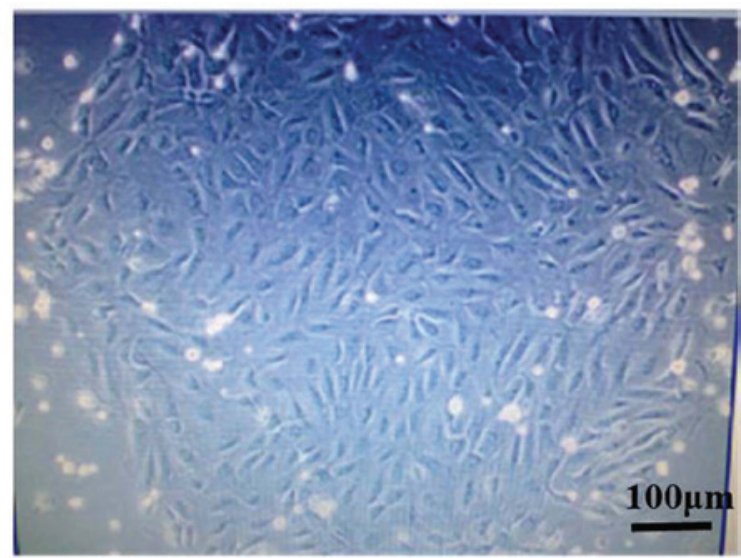

$\mathrm{D}$

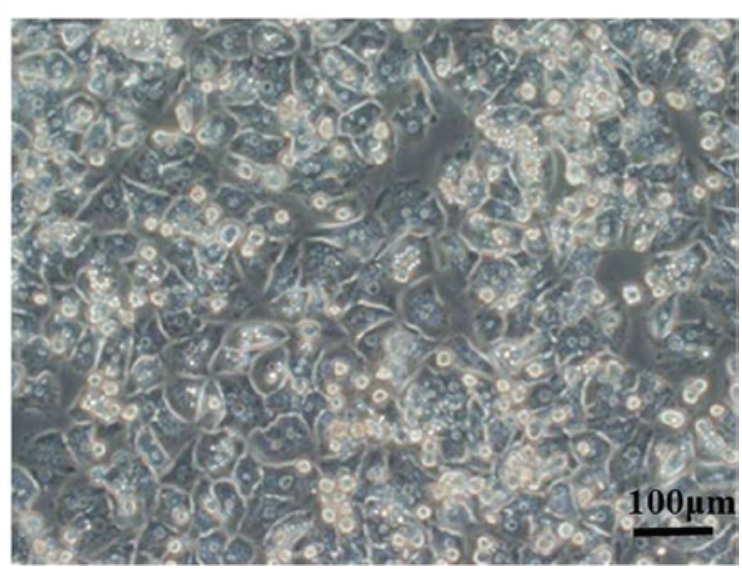

Figure 5. Isolated human hepatocellular adenoma cells were observed under optical microscopy and representative images present the cell morphology after: (A) $24 \mathrm{~h}$ of culture; (B) $72 \mathrm{~h}$ of culture; and (C) the first passage. (D) Images of primary normal liver cells as a control.

morbidity and mortality. BAL systems aim to serve as a potential supportive therapy for patients with liver failure that are waiting for or recovering from transplantation (7). However, the development of a BAL has been impeded by the scarcity of suitable biological material (21). In the present study, the feasibility of HCA tissue as a cellular source for BAL systems was assessed. The results demonstrated a greater prevalence of HCA in females compared with males, which is consistent with results of previous reports (22). Histological examination demonstrated that hepatocytes in HCA tissue exhibited no obvious atypia and possessed similar morphology to that of hepatocytes in healthy liver tissues. Histological analysis therefore revealed that HCA tissues may function in a similar manner to healthy liver tissues.

As a crucial component of BAL, many tumor-derived human cell lines have been studied as potential biological material for BAL models. The $\mathrm{C} 3 \mathrm{~A}$ cell line, as a clonal derivative of HepG2 cells, has been used in the extracorporeal liver assist device system to treat patients with ALF (23). However, no significant effect on survival was observed due to poor ammonia detoxification and mixed function oxidase activity (24). The HepaRG cell line has been reported to be a suitable model for primary human hepatocytes with respect to retaining various hepatic functions following culture for a 14-day proliferation phase in HepaRG medium then a 14-day differentiation phase with $2 \%$ dimethylsulfoxide (DMSO). In 2011, Hoekstra and Chamuleau (25) alluded to the potential of these cells in BAL, based on their high metabolic and synthetic functionality, but no further clinical research involving these cells has been reported. Until now, to the best of our knowledge, no human cell lines have been demonstrated to be suitable for application in BAL, primarily due to insufficient functionality.

HCA is a benign liver tumor and its monoclonal properties may facilitate cell lineage establishment and storage. In the current study, experiments were performed to determine whether HCA has potential to be a cell source for BAL development. The expression of genes involved in various hepatic functions were analyzed using PAS staining, IHC and RT-qPCR in HCA tissues and corresponding adjacent liver tissues. Importantly, the expression of synthetic function-specific genes (ALB, TTR, AAT and TF), detoxification-related genes (CYT2E1, CYT3A5 and CYT3A4), as well as the genes related to metabolism and conversion (GST- $\alpha$, CPS-1, TAT) demonstrated no statistical difference between HCA and adjacent liver tissues, although a larger sample size is needed for confirmation of results. In addition, according to RT-qPCR data, AFP expression was increased in HCA compared with healthy liver tissues. Notably, the expression of AFP in HCA and normal tissues were very low and considerably less than that observed in HCC tissues in a previous study by the current authors (data not published). Exploration of the functionality of HCA tissues provides early evidence of this potential, indicating that further research into the possibility of using these tissues as a source for the biological component of BAL is warranted. However, the current study is preliminary and future research should focus 
on the establishment of an HCA cell line and confirmation of its functional abilities.

In addition to functionality, the proliferative capability of cells is also crucial for their application in a BAL system in clinical practice. For BAL development, primary human hepatocytes are an ideal source of biological material due to their high level of liver function. However, 10 billion fully functional primary hepatocytes are required for each BAL and primary cells are scarce and cannot be efficiently expanded in vitro (26). Therefore, a cell line with the functionality of primary hepatocytes is desired. In the present study, isolating primary cells from HCA tissues proliferated for weeks in vitro. Given the monoclonal characteristics of HCA, the superiority in the proliferative ability of HCA-derived cells and the greater potential for cell line preservation compared with normal hepatocytes, HCA tissues may be a promising alternative to human hepatocytes as a source of the cellular component of BAL systems (13). It is possible that cells with higher proliferative potential were deliberately selected and that these cells exhibited more pronounced characteristics of cancer than the non-selected cells. Mutation of proliferation-associated genes may have occurred in the active cell colony. Thus, the safety issues involved in the use of HCA remain unclear which is the limitation of the current study. As a result, safety evaluation should be performed in future study after the cell lineage is established.

With their superior proliferative ability and comparable liver-specific function relative to primary hepatocytes, HCA-derived cells represent a potentially ideal cell source for a BAL. Future work will focus on cell lineage establishment. Furthermore, the safety issues regarding the use of HCA remain unclear and require more extensive and specific investigation.

\section{Acknowledgments}

Not applicable.

\section{Funding}

The current study was supported by the Science and Technology Planning Project of Guangdong Province (grant no. 2014A050503041), the Science and Technology Planning Project of Guangzhou (grant no. 2014J4500013), and the Guangdong Provincial Bioengineering Research Center for Gastroenterology Diseases (grant no. 2017B02029003).

\section{Availability of data and materials}

The datasets used and/or analyzed during the current study are available from the corresponding author on reasonable request.

\section{Authors' contributions}

AL conceived the current study and provided technical support. QY and XZ performed the experiments. QY and LD collected the specimens. YF collected the data and performed statistical analysis. QY and JL drafted the manuscript. All authors read and approved the final manuscript.

\section{Ethics approval and consent to participate}

The Medical Ethics Committee of Nanfang Hospital, Southern Medical University (Guangzhou, China) approved all of the procedures performed in this study. All enrolled subjects provided their written informed consent.

\section{Patient consent for publication}

Not applicable.

\section{Competing interests}

The authors declare that they have no competing interests.

\section{References}

1. Demetriou AA: Support of the acutely failing liver: State of the art. Ann Surg 228: 14-15, 1998.

2. Leventhal TM and Liu KD: What a nephrologist needs to know about acute liver failure. Adv Chronic Kidney Dis 22: 376-381, 2015.

3. Bernal W, Hyyrylainen A, Gera A, Audimoolam VK, McPhail MJ, Auzinger G, Rela M, Heaton N, O'Grady JG, Wendon J and Williams R: Lessons from look-back in acute liver failure? A single centre experience of 3300 patients. J Hepatol 59: 74-80, 2013.

4. Hu X, Yang T, Li C, Zhang L, Li M, Huang W and Zhou P: Human fetal hepatocyte line, L-02, exhibits good liver function in vitro and in an acute liver failure model. Transplant Proc 45: 695-700, 2013.

5. Guoliang L, Anye Z, Lifu Z, Xiaoping P, Yimin Z, Chengbo Y, Yuemei $C$ and Lanjuan L: Effects of plasma from acute-on-chronic liver failure patients on immortalized human hepatocytes in vitro. Hepatogastroenterology 58: 1328-1333, 2011.

6. Punzalan CS and Barry CT: Acute liver failure: Diagnosis and management. J Intensive Care Med 31: 642-653, 2016.

7. Li Y, Wu Q, Wang Y, Weng C, He Y, Gao M, Yang G, Li L, Chen F, Shi Y, et al: Novel spheroid reservoir bioartificial liver improves survival of nonhuman primates in a toxin-induced model of acute liver failure. Theranostics 8: 5562-5574, 2018.

8. Filippi C, Keatch SA, Rangar D, Nelson LJ, Hayes PC and Plevris JN: Improvement of C3A cell metabolism for usage in bioartificial liver support systems. J Hepatol 41: 599-605, 2004.

9. Bonavita AG, Quaresma K, Cotta-de-Almeida V, Pinto MA, Saraiva RM and Alves LA: Hepatocyte xenotransplantation for treating liver disease. Xenotransplantation 17: 181-187, 2010.

10. Demetriou AA, Brown RS Jr, Busuttil RW, Fair J, McGuire BM, Rosenthal P, Am Esch JS II, Lerut J, Nyberg SL, Salizzoni M, et al: Prospective, randomized, multicenter, controlled trial of a bioartificial liver in treating acute liver failure. Ann Surg 239: 660-670, 2004

11. Shi XL, Han B, Tan JJ, Yuan X, Zhang Y, Xiao JQ, Gu ZZ and Ding YT: Factors influencing the transfer of porcine endogenous retroviruses across the membrane in bioartificial livers. Int $\mathbf{J}$ Artif Organs 35: 385-391, 2012.

12. Farges O, Ferreira N, Dokmak S, Belghiti J, Bedossa P and Paradis V: Changing trends in malignant transformation of hepatocellular adenoma. Gut 60: 85-89, 2011.

13. Gouw AS, Zeng W, Buiskool M, Platteel I, van den Heuvel MC, Poppema S, de Jong KP and Molema G: Molecular characterization of the vascular features of focal nodular hyperplasia and hepatocellular adenoma: A role for angiopoietin-1. Hepatology 52: 540-549, 2010.

14. Ji C, Annabi N, Khademhosseini A and Dehghani F: Fabrication of porous chitosan scaffolds for soft tissue engineering using dense gas CO2. Acta Biomater 7: 1653-1664, 2011.

15. Kunstman JW, Korah R, Healy JM, Prasad M and Carling T: Quantitative assessment of RASSF1A methylation as a putative molecular marker in papillary thyroid carcinoma. Surgery 154: 1255-1262, 2013

16. Livak KJ and Schmittgen TD: Analysis of relative gene expression data using real-time quantitative PCR and the 2(-Delta Delta C(T)) method. Methods 25: 402-408, 2001. 
17. Selden C, Chalmers SA, Jones C, Standish R, Quaglia A Rolando N, Burroughs AK, Rolles K, Dhillon A and Hodgson HJ: Epithelial colonies cultured from human explanted liver in subacute hepatic failure exhibit hepatocyte, biliary epithelial, and stem cell phenotypic markers. Stem Cells 21: 624-631, 2003

18. Tonorezos ES, Barnea D, Abou-Alfa GK, Bromberg J, D'Angelica M, Sklar CA, Shia J and Oeffinger KC: Hepatocellular adenoma among adult survivors of childhood and young adult cancer. Pediatr Blood Cancer 64: e26294, 2017.

19. Vijay A, Elaffandi A and Khalaf H: Hepatocellular adenoma: An update. World J Hepatol 7: 2603-2609, 2015.

20. Lee SY, Kim HJ and Choi D: Cell sources, liver support systems and liver tissue engineering: Alternatives to liver transplantation. Int J Stem Cells 8: 36-47, 2015.

21. Kandiah PA and Subramanian RM: Extracorporeal devices. Crit Care Clin 35: 135-150, 2019.

22. Khanna M, Ramanathan S, Fasih N, Schieda N, Virmani V and McInnes MD: Current updates on the molecular genetics and magnetic resonance imaging of focal nodular hyperplasia and hepatocellular adenoma. Insights Imaging 6: 347-362, 2015.
23. Ellis AJ,Hughes RD, Wendon JA, Dunne J,Langley PG, Kelly JH, Gislason GT, Sussman NL and Williams R: Pilot-controlled trial of the extracorporeal liver assist device in acute liver failure. Hepatology 24: 1446-1451, 1996.

24. Chamuleau RA, Deurholt T and Hoekstra R: Which are the right cells to be used in a bioartificial liver? Metab Brain Dis 20: 327-335, 2005.

25. Hoekstra R and Chamuleau RA: Recent developments on human cell lines for the bioartificial liver. Int J Artif Organs 25: 182-191, 2002.

26. Hoekstra R, Nibourg GA, van der Hoeven TV, Ackermans MT, Hakvoort TB, van Gulik TM, Lamers WH, Elferink RP and Chamuleau RA: The HepaRG cell line is suitable for bioartificial liver application. Int J Biochem Cell Biol 43: 1483-1489, 2011. 